\title{
Supporting the Living of the Elderly with Semantic Collaborative HealthCare
}

\author{
Haipeng Wang, Xingshe Zhou, Zhu Wang \\ School of Computer Science \\ Northwestern Polytechnical University \\ Xi'an, P.R.China \\ Email: \{haipeng, zhouxs\}@nwpu.edu.cn
}

\begin{abstract}
Nowadays, for supporting the independent living and emergency treatment of the elderly, a number of individuals (e.g., professionals, caregivers, relatives, neighbors, and friends) have been involved in the healthcare process. The collaboration among them can supply more information to help making correct decision and taking action timely. However, it is a challenge to achieving an effective collaborative healthcare among multiple parities with individual knowledge, located in different places, operating in different time, and doing not know each other even. For addressing the challenges in collaborative healthcare, this paper proposes an ontology-based semantic collaborative healthcare approach to supporting and enhancing the living of the elderly people. This approach achieves the semantic collaboration by exploiting the knowledge about the elderly, healthcare related information tuple, and the users including professional, relatives and so on. Ontology is utilized to model and represent such kinds of knowledge. A tuplespace-based coordination model is adopted to support the cooperation among different parities at anytime and anywhere with anybody. We built a prototype system, and evaluated its effectiveness with preliminary experiments.
\end{abstract}

Keywords-collaborative healthcare; semantic tuplespace; ontology; adaptive information presentation

\section{INTRODUCTION}

Nowadays, the provision of healthcare typically involves a number of individuals, located in different institutions. With the effective cooperation among them, more information may be supplied to the professionals or caregivers for make accurate decision and take timely action. For example, as an elderly person falls down, the professionals are able to make correct decision based on the text notification associated with the video taken on scene of action. Furthermore, the relatives, neighbors, and the friends may also provide supplementary action to the emergency treatment, as the professionals and caregivers are not available. For the former example, the neighbor may be the best person to take timely action to deal with that emergency case. So the collaborative healthcare can not only provide effective healthcare treatment, but also reduce the cost spent on the whole system[1].

For effective collaborative healthcare, the supporting system should take into account the contextual information of the users (e.g., the elderly person's disabilities and relatives, relatives' current activities, and professionals' access terminals.) and operate in semantic layer for understanding the meaning of information. We call this new paradigm of healthcare as

This research is supported by the National High Technology Development 863 Program of China under Grant No. 2006AA01Z198 and the Innovation Fund of Northwestern Polytechnical University of China under Grant No. 2006CR13.

Permission to make digital or hard copies of all or part of this work for personal or classroom use is granted without fee provided that copies are not made or distributed for profit or commercial advantage and that copies bear this notice and the full citation on the first page. To copy otherwise, to republish, to post on servers or to redistribute to lists, requires prior specific permission and/or a fee. PERVASIVEHEALTH 2008, 30 Jan - 1 Feb. Tampere, Finland

Copyright $(9) 2008$ ICST 978-963-9799-15-8

DOI 10.4108/ICST PERVASIVEHEALTH2008.2531 semantic collaborative healthcare. In this paper, we propose an ontology-based approach for semantic collaborative healthcare to realize the effective semantic cooperation in healthcare for the elderly people. We address the semantic collaboration by exploiting the knowledge about the elderly, the healthcare information, and the users including the professionals, caregivers, relatives and so on. We adopt the tuplespace-based collaborative model to support the cooperation among different parties [7]. This approach is characterized with time decoupling, space decoupling, and name free features. The knowledge modeling and whole semantic collaborative process are performed based on ontology.

The rest of the paper is organized as follows: Section 2 discusses previous work relevant to this paper. In section 3 , we present the ontology model to express knowledge about the elderly, healthcare related information tuple, and the user. Section 4 describes the semantic collaborative healthcare in detail. The prototype implementation and preliminary results are presented in section 5. Finally, Section 6 concludes the paper.

\section{RELATED WORK}

Several works have been reported in the past years to address the collaboration topic in the healthcare. SuperAssist [2] is a user-assistant collaborative environment for the supervision of medical instrument use at home. It aims to improve the performance of maintenance and trouble shooting. It proposes to perform collaboration of users of medical instruments with online assistants, cooperative assistants, and autonomous assistants to achieve its goal.

Ruiz et al. [3] discuss the collaboration topic in ubiquitous diagnosis. It is mainly based on the web interface and internetenabled technology to support remote and immediate initial diagnosis in case a medical specialist is not available. This work shows that the collaboration is an effective approach to addressing the distributed knowledge management problems in healthcare. Bravo et al. [4] address the topic of informal meetings among doctors and nurses. It adopts the RFID technology for improving the collaboration between the user and the computer. For example, it combines the information coming from patients' records in the hospital data base with the private and more specific information included in the individual doctors' or nurses' tags. Hassinen et al. [5] concern the coordination in Emergency Medical Services (EMS), especially in coordinating disaster relief effort. It utilizes a number of portable devices, such as TablePC, barcoder reader, 
and digital pen, to collect and send data among multiple parties, which facilitates the data inputting and gathering effectively.

Our work differs from previous work in several aspects. First, we propose semantic collaborative healthcare, which is achieved based on the elderly and information ontologies. Second, we aim to support the multiple parties' coordination in anytime, anywhere with anybody based on a tuplespace coordination model. Finally, we integrate the mechanism of adaptive information notification into the collaborative healthcare for achieving context-awareness information presentation, which is important for emergency treatment.

\section{ONTOLOGY MODEL}

We use the ontologies to model knowledge about the elderly, healthcare information, and the users including the professionals, caregivers, relatives and so on. Within the domain of knowledge representation, term ontology refers to the formal and explicit description of domain concepts, which are often conceived as a set of entities, relations, instances, functions, and axioms[6]. By allowing individuals and healthcare related information to share a common understanding of knowledge structure, the ontologies enable applications to interpret the elderly and other users context and healthcare information features based on their semantics. Furthermore, ontologies' hierarchical structure lets developers reuse domain ontologies (e.g., of body functions, body impairments, etc.) in describing the elderly states and build a practical model without starting from scratch.

In our system, we have design three ontologies: Elderly Ontology, Information Tuple Ontology, and User Ontology. The elderly ontology, shown in fig.1, depicts contexts about the elderly, which mainly includes the disabilities the elderly suffered (for example, the hearing or seeing impairments), and the relationship the elderly have (for example, their professionals, caregivers, relatives, and neighbors). We use the pairs of feature and degree to describe these disabilities, in which the feature refers to certain disability, and the degree describes its degree with high, middle and low. For example, the pair of (hearing, high) describes the hearing impairment of the elderly is in high degree. The information tuple ontology, shown in fig. 2, depicts the properties of healthcare information, which are formalized in the notion of tuple[7]. InfoTuple has the feature of priority, which indicates that the information tuple is emergent (e.g., fall down case), regular (e.g., sign of heartbeat or blood), or normal (e.g., medicine taking). AccessRight indicates that who has the right to access the information tuple. Currently, the right includes the read, take, and put, corresponding to reading, deleting, and generating a information tuple respectively. The User Ontology, shown in fig. 3, depicts the features of users related the elderly (e.g., their professionals, caregivers, relatives, and so on), which mainly includes the user's role associated with access rights to certain information tuple, the terminal which user may used to access information, and the contextual information, e.g., location, time, and activity, and so on. $u o$ and eo stand for User Ontology and Elderly Ontology respectively.

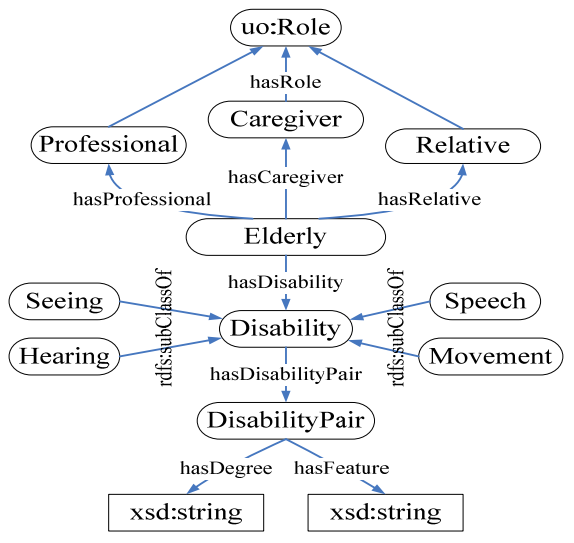

Figure 1. Elderly ontology

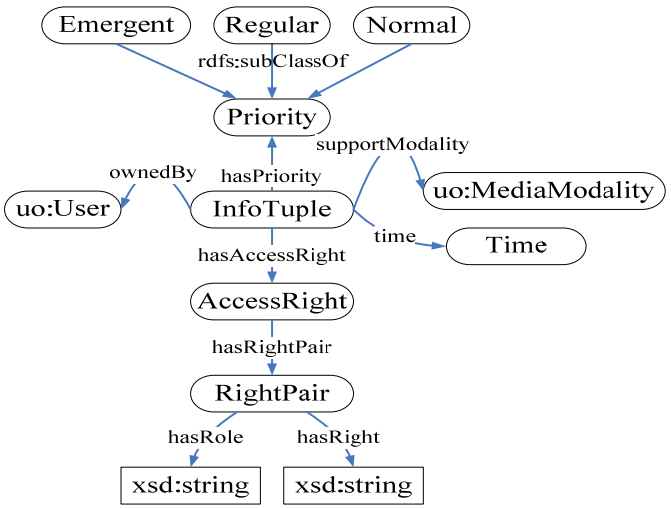

Figure 2. Information Tuple ontology

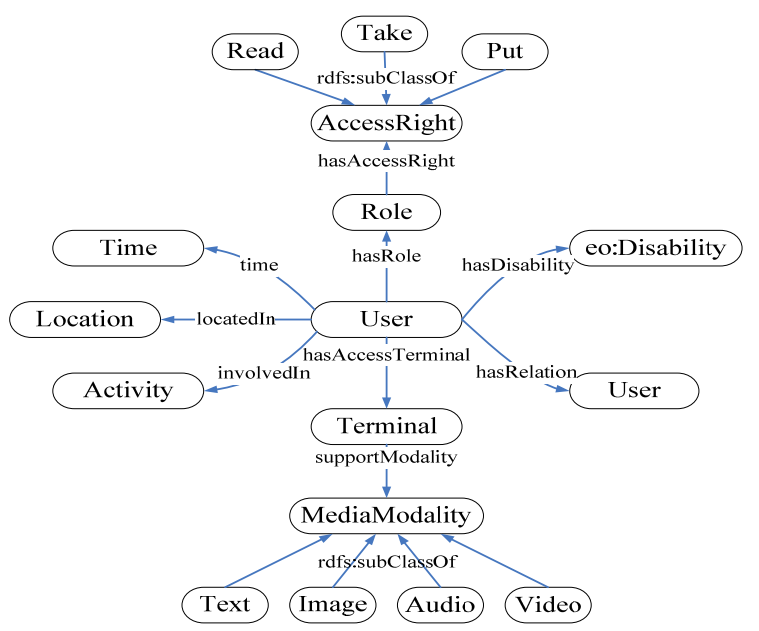

Figure 3. User ontology

We adopt OWL (Web Ontology Language)[8] to express ontology enabling expressive knowledge description and data interoperability of knowledge. It mainly includes ontology class definition and ontology instance markups to describe the three ontologies about elderly, information tuple, and user defined above. 


\section{Collaborative HeAlcare}

For achieving the collaborative healthcare, we firstly adopt the RBM (role-based management) approach to organizing all the persons involved in the healthcare process, and assign each person with distinct authorization and obligation policies. Based on the role-based user management, we adopt the tuplespace-based collaboration model to orchestrate the actions of all users. The tuple is used to model the healthcare information, and all the actions issued by users are classified into three categories, including read, take, and put information tuple. Finally, adaptive information notification provides the mechanism to present the suitable information to users in a personalized and context-awareness manner.

\section{A. Role-Based User Management}

With more individuals involved in the elderly healthcare, it needs the support for interaction among multiple parties. The theory of role is an effective method to address this topic. Rolebase management (RBM) is proposed in [9] for managing distributed systems. A role is defined as a collection of rights and duties, which correspond to authorization and obligation policies respectively. Users assigned to certain roles have corresponding access right and were required to fulfill the role obligations. Roles may be organized into a hierarchy that enables policies to be inherited across roles thus facilitating policy reuse. So we can use the roles to describe all the users and services involved in the healthcare systems.

We have designed several kinds of roles to define the users involved in the healthcare systems. Each role has the access right to deal with the healthcare information about the elderly. The access rights include read, put, and take right applied on the information tuple, which means that the role can read, generate and take or delete healthcare related information about the elderly respectively. This design principle is related to the choice of tuplespace-based collaborative model, described later. The roles are described as follows:

1) elderly: This role refers to the elderly themselves. This role has the access rights of read, put, and take to information. This means that the elderly person can find out information about their body provided by sensors, can put new information about their healthcare states, and delete obsolete information after the treatment of emergency cases.

2) professional: This role refers to the elderly person's professionals. Since they have the knowledge to make decision and take actions, so this role has the access rights to read, put, and take information about the elderly. For example, upon receiving the reminder of emergency case, they are able to put new information to inform the relatives and caregivers to take action immediately.

3) caregiver: This role refers to the elderly person's caregivers. This role has the access rights of read, put, and take information about the elderly.

4) relative: This role refers to elderly person's relatives. This role has the access rights of read and put information about the elderly.
5) others: This role includes the elderly person's neighbors, friends and so on. This role has the access right of read to get related information of the elderly.

The definition of roles' access rights is only a default and static case; on the other hand, the system can assign and adjust roles' access rights dynamically. For example, upon receiving the emergency reminder, the professionals may create a new information to inform the caregivers and relatives to perform different actions by assigning different access rights, i.e., assign that the caregivers and relatives both have the read right to be notified of this reminder, but only the caregivers have the take right to delete this information after fulfill professional treatment for such emergency case. If the creator of information does not specify the access rights, the system will take the default values. By adopting the role-base management, we can achieve the effective management all users and services involved in the healthcare systems.

\section{B. TupleSpace-Based Collaborative Healthcare Model}

We adopt the tuplespace-based coordination model to support the collaborative healthcare for the elderly. The tuplespace paradigm is a simple and efficient approach for supporting cooperative communication among distributed entities. Typically, a tuplespace system contains three major components: (1) tuple writers, who write or put tuples into sharespace, (2) tuple readers, who read/take tuples that they are interested in, by specifying templates, and (3) the tuplespace server, who is responsible for managing the sharespace and routing the tuples from writers to readers. The earliest tuplespace systems were type-based, and only enable type matching. Recently, semantic tuplespaces systems have been developed to support the semantic tuples matching [10]. Based on this feature and the ontologies, developed in this paper, it is possible for different parities to understand the meaning of different healthcare cases.

In the tuplespace-based collaborative model, used in our system, multiple users perform the elderly-centered healthcare by reading, putting (writing), or taking related information tuple from tuplespace. Each user plays certain role associated with a set of access rights. By take the time and space decoupling and name-free features, provided by tuplespace, the different parties, including the professionals, caregivers, relatives, and others, are able to carry out collaborative healthcare for the elderly at anytime, anywhere, with anybody; the persons even do not know each other in advances. For example, by investigating the data of the elderly, the professional may adjust the schedule of medicine taking, and write an updated plan into tuplespace to inform the caregivers and relatives. In this example, the professionals and caregivers do not need to know each other for cooperating in same time or place; furthermore, they do not need to know the existence of each other.

The information tuple ontology is used to provide information classification (i.e., emergent, regular, and normal), healthcare treatment authorization (e.g., an emergency information tuple can specify that only professionals and caregivers have the right to deal with), and adaptive information notification (described in next subsection). 


\section{Adaptive Information Notification}

Adaptive information notification refers to present the healthcare information to right person, at right time and situation, and in an appropriate way. For example, an emergence case information should sent to the elderly person's professionals and caregivers, and present in text and video way for professionals with the large display screens, and present in speech-based warning way for the caregivers equipped with PDAs in driving mode.

To achieve adaptive information notification, the Elderly Ontology, Information Tuple ontology, and User Ontology play a central role. In the aforementioned example, the emergency case information about the elderly is sent to the right person, such as their relatives, by exploiting the Elderly Ontology. It is sent at right time and situation by exploiting the User Ontology, and is sent in appropriate manner by exploiting Information Tuple Ontology and User Ontology.

Adaptive information notification plays an important role in improve the accuracy and timely of healthcare for the elderly. For example, as the elderly fall down, the presentation of this emergency treatment information in both text and video way is helpful for professionals to make correct decision, and is important to present in speech way for relatives to get timely notification as they are in driving car mode.

\section{PROTOTYPE IMPLEMENTATION AND EXPERIMENT}

With the proposed collaborative HealthCare approach, we built a prototype system and carried out the preliminary experiments. We adopt the JavaSpace as the tuplespace platform with the extension in semantic tuple matching support and developed the overall system with Java. An experiment of the emergency treatment about the elderly falling down is designed.

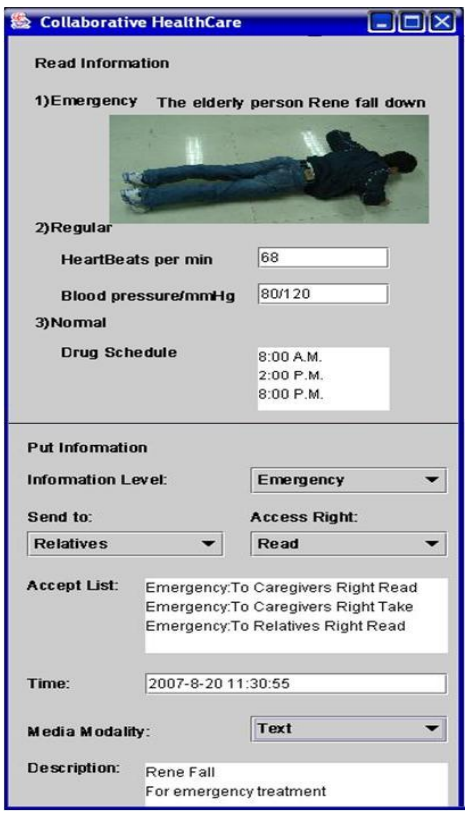

(a)

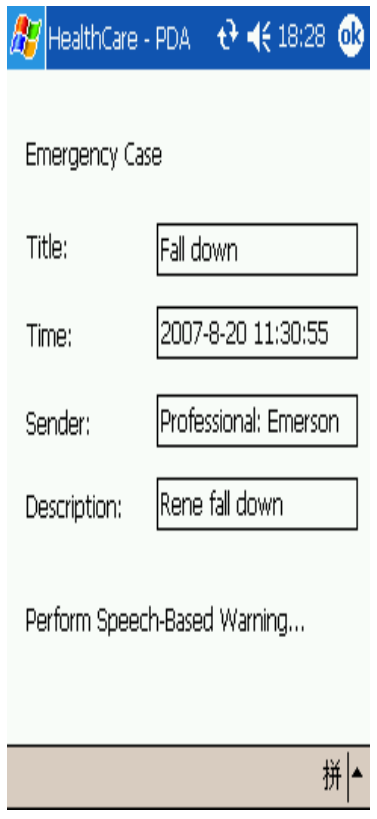

(b)
Figure 4. Client-side interface for collaborative healthcare for the elderly
Fig. 4 shows several client-side interfaces of the prototype system. Fig. $4 \mathrm{a}$ is the main interface presented to the elderly person's professionals with a PDP displayer (a large plasma display panel) in their offices. It mainly consists of two parts. The upper side part provides interface to acquire healthcare information, and presents in adaptive manner. For example, with the support of device capabilities, the information about this emergency case is able to present in both text and video manner, which is help to make correct decision for professionals. The lower side part is the interface to edit and send new information tuple to certain persons. In this example, as the professionals make the decision about the elderly person's fall down, they compose a new information tuple to inform the caregivers and relatives to provide help or take action. Fig. $4 \mathrm{~b}$ is the interface presented to the elderly person's relatives in their PDA terminals. As the relatives are in driving car mode, so this information is presented in speech manner for avoiding visual conflict and resulting in a safe notification.

\section{CONCLUSION AND FUTURE WROK}

As more people have been involved in the healthcare for the elderly, there is a need to provide the support for coordinating the behaviors of all individuals. To fulfill this requirement and address related challenges, this paper presents a semantic collaborative healthcare approach. This approach has two distinct features: it provides an ontology-based semantic collaboration, and it supports the cooperation among different persons in anytime, anywhere, and with anybody based on a tuplespace-based coordination model. Finally, we built a prototype system, and the preliminary experiments show its effectiveness in the emergency treatment of the elderly.

\section{REFERENCES}

[1] Rose-Marie Droes, et al., "Healthcare Systems and Other Applications," IEEE Pervasive Computing, vol. 6, pp. 59-63, Jan. 2007.

[2] Olivier A. Blanson Henkemans, et al., "SuperAssist: A User-Assistant Collaborative Environment for the supervision of medical instrument use at home," in proceedings of the 1st International Conference on Pervasive Computing Technologies for Healthcare, Austria, Nov. 2006.

[3] Daniel Ruiz, et al., "Ubiquitous Diagnosis: Assurance through Distribution and Collaboration," in proceedings of the 1st International Conference on Pervasive Computing Technologies for Healthcare, Austria, Nov. 2006.

[4] J. Bravo, et al., "Supporting Informal Meetings in Hospitals," in proceedings of the 1st International Conference on Pervasive Computing Technologies for Healthcare 2006, Innsbruck, Austria, November, 2006.

[5] Marko Hassinen, Maija Marttila-Kontio, "Disaster Relief Coordination using a Documentation System for Emergency Medical Services,” in proceedings of the 1st International Conference on Pervasive Computing Technologies for Healthcare 2006, Innsbruck, Austria, November, 2006.

[6] T. Gruber, "A Translation Approach to Portable Ontology Specification," Knowledge Acquisition, vol. 5, no.2, pp.199-220, 1993.

[7] David Gelernter, "Generative Communication in Linda," ACM Transactions on Programming Languages and Systems (TOPLAS), vol 7, no. 1, pp. 80-112, 1985 .

[8] D. L. McGuinness, F. Harmelen, "OWL Web Ontology Language Overview,” W3C Recommendation, 2004

[9] E.Lupu, “ A Role-Based Framework for Distributed Systems Management," PhD Thesis, Imperial College, London, 1998.

[10] Deepali Khushraj, et al., "sTuples: Semantic Tuple Spaces," in Proceedings of the 1st Annual International Conference on Mobile and Ubiquitous Systems: Networking and Services, Boston, pp. 268-277, 2004. 